International Mathematical Forum, 1, 2006, no. 1, 47-49

\title{
Coverings of curves and non-existence of certain models of them in the quadric surface
}

\author{
E. Ballico ${ }^{1}$ \\ Dept. of Mathematics, University of Trento \\ 38050 Povo (TN), Italy \\ ballico@science.unitn.it
}

\begin{abstract}
Let $C \subset S:=\mathbf{P}^{1} \times \mathbf{P}^{1}$ be an integral curve and $\nu: X \rightarrow C$ its normalization. Set $L:=\nu^{*}\left(\mathcal{O}_{S}(1,0)\right)$. Here we give numerical conditions which imply that the pair $(X, L)$ is not the pull-back of a pair $(Y, R)$ with $Y$ a smooth curve of genus $q>0$ and $R \in \operatorname{Pic}(Y)$.
\end{abstract}

Mathematics Subject Classification: 14H50; 14H51

Keywords: coverings of curves; quadric surface; space curve

\section{INTRODUCTION}

Let $X$ be a smooth and connected projective curve. There are many high degree pencils $f_{i}: X \rightarrow \mathbf{P}^{1}, i=1,2$, such that the induced map $\left(f_{1}, f_{2}\right): X \rightarrow S:=\mathbf{P}^{1} \times \mathbf{P}^{1}$ is birational onto its image. However, very seldom there are such morphisms for which the image $\left(f_{1}, f_{2}\right)(X)$ has "very few" singularities. Here we prove the following quantitative versions of this observation.

Theorem 1. Fix integer $u, v, a$ such that $v \geq u \geq 4$ and $0 \leq a \leq(u+1)(v+1)-3 a-$ 4. Fix a general $A \subset S:=\mathbf{P}^{1} \times \mathbf{P}^{1}$ such that there $\sharp(A)=a$. Let $\Gamma_{A}$ denote the set of all integral and nodal curves $C \subset\left|\mathcal{O}_{S}(u, v)\right|$ such that $\operatorname{Sing}(C)=A$. The set $\Gamma_{A}$ is a non-empty open subset of an $(u v+u+v-3 a)$-dimensional projective space. Fix a general $C \in \Gamma_{A}$ and let $\nu: X \rightarrow C$ be the normalization. Then $X$ is not a multiple covering $f: X \rightarrow Y$ of a smooth curve of genus $>0(\operatorname{deg}(f)>1)$ such that there is $R \in \operatorname{Pic}(Y)$ with $\left.\nu^{*}\left(\mathcal{O}_{C}(1,0)\right) \cong f^{*}(R)\right)$ and $h^{0}\left(X, \nu^{*}\left(\mathcal{O}_{C}(1,0)\right)\right)=h^{0}(Y, R)$.

Theorem 2. Fix integers $m, k, q, v, a$ such that $k \geq 2, m \geq 2, q>0, v \geq m k$ and $0 \leq a \leq \min \{m k-1, v-2-\lceil(q+1) / m\rceil\}$. Let $C \in\left|\mathcal{O}_{S}(m k, v)\right|, S:=\mathbf{P}^{1} \times \mathbf{P}^{1}$, be any integral curve such that the conductor of the normalization $\nu: X \rightarrow C$ has length $a$. Then $X$ is not a degree $k$ multiple covering $f: X \rightarrow Y$ of a smooth curve of genus $q$ such that there is $R \in \operatorname{Pic}^{m}(Y)$ with $\left.\nu^{*}\left(\mathcal{O}_{C}(1,0)\right) \cong f^{*}(R)\right)$ and $h^{0}\left(X, \nu^{*}\left(\mathcal{O}_{C}(1,0)\right)\right)=h^{0}(Y, R)$.

We work over an algebraically closed field $\mathbb{K}$ with $\operatorname{char}(\mathbb{K})=0$.

\footnotetext{
${ }^{1}$ The author was partially supported by MIUR and GNSAGA of INdAM (Italy)
} 
Example 1. Set $S:=\mathbf{P}^{1} \times \mathbf{P}^{1}$. Let $\pi_{1}: S \rightarrow \mathbf{P}^{1}$ and $\pi_{2}: S \rightarrow \mathbf{P}^{1}$ denote the two projections. We have $\operatorname{Pic}(S) \cong \mathbb{Z}^{\oplus 2}$ with, as generators, the class $\mathcal{O}_{S}(1,0)$ of a fiber of $\pi_{1}$ and he class $\mathcal{O}_{S}(0,1)$ of a fiber of $\pi_{2}$. For all $P \in S$ let $2 P$ denote the first infinitesimal neighborhood of $P$ in $S$, i.e. the closed subscheme of $S$ with $\mathcal{I}_{P, S}{ }^{2}$ as its ideal sheaf. Hence $(2 P)_{\text {red }}\{P\}$, and length $(2 P)=3$ and length $(3 P)=6$. Fix any integral curve $C \in\left|\mathcal{O}_{S}(u, v)\right|$ and set $A(C):=\operatorname{Sing}(C)$. Let $\nu: X \rightarrow$ $C$ be the normalization map and $B(C) \subset S$ the conductor of $X$ in $S$. Hence $p_{a}(X)=u v-u-v+1-\operatorname{length}(B(C))$. We recall that $B(C)=A(C)$ if $C$ has only ordinary nodes and ordinary cusps as its singularities. Since $\omega_{S} \cong \mathcal{O}_{S}(-2,-2)$, we have $h^{0}\left(S, \omega_{S}\right)=h^{1}\left(S, \omega_{S}\right)=0$. Hence the classical adjunction theory developed for plane curves works for curves in $S$ and gives $H^{0}\left(X, \omega_{X}\right) \cong H^{0}\left(S, \mathcal{I}_{B(C)}(u-2, v-2)\right)$. Set $L:=\nu^{*}\left(\mathcal{O}_{C}(1,0)\right)$. Hence $L$ is a degree $u$ spanned line bundle on $X$. Fix an integer $k$ such that $1 \leq k \leq v-3$. By adjunction theory we have $h^{0}\left(X, L^{\otimes k}\right)=$ $k+1+h^{1}\left(S, \mathcal{O}_{S}(u-2, v-2-k)\right)$.

Proof of Theorem 1. The non-emptiness of $\Gamma_{A}$ and its dimension easily follow from [1], Cor. 4.6, because $\omega_{S}{ }^{*}$ is ample. Set $Z:=\bigcup_{P \in A} 2 P$. By [1], Cor. 4.6, we have $h^{1}\left(S, \mathcal{I}_{Z}(u, v)\right)=0$. Since $P$ is general in $S$ and $h^{0}\left(S, \mathcal{I}_{Z}(u, v)\right) \neq 0$, we get $h^{1}\left(S, \mathcal{I}_{Z \cup\{P\}}(u, v)\right)=0$. Fix a general $T \in\left|\mathcal{O}_{S}(1,0)\right|$ and a general $P \in T$. Hence $T \cap A=\emptyset$. For any quasi-projective scheme $\Theta$ and any $Q \in \Theta_{\text {reg }}$ let $\{2 Q, \Theta\}$ denote the first infinitesimal neighborhood of $Q$ in $\Theta$. Hence $\{2 P, T\}$ is the degree two closed subscheme of $T$ with $P$ as support. Set $Z^{\prime}=Z \cup\{2 P, T\}$. Take another general $P^{\prime} \in T$ and set $Z^{\prime \prime}:=Z^{\prime} \cup\left\{2 P^{\prime}, T\right\}$.

First Claim: $h^{1}\left(S, \mathcal{I}_{Z^{\prime}}(u, v)\right)=0$.

Proof of the First Claim: Set $W:=\bigcup_{Q \in \pi_{1}(A)}\left\{2 Q, \mathbf{P}^{1}\right\}$. Assume $h^{1}\left(S, \mathcal{I}_{Z^{\prime}}(u, v)\right) \neq$ 0 . Since $h^{0}\left(S, \mathcal{I}_{Z}(u, v)\right) \geq 2$ and $P$ is general in $S$, we get that the differential of the rational map induced on $S$ by linear system $\left|\mathcal{I}_{Z}(u, v)\right|$ vanishes identically in the direction of the $\pi_{1}$-fibering. Since $\operatorname{char}(\mathbb{K})=0$, this implies that this rational map factors through $\pi_{1}$. Hence $h^{0}\left(S, \mathcal{I}_{Z}(u, v)\right)=h^{0}\left(\mathbf{P}^{1}, \mathcal{I}_{W}(v)\right)=\max \left\{0, v+1-\sharp\left(\pi_{1}(A)\right)\right\}$. Since $\sharp\left(\pi_{1}(A)\right)=0, h^{0}\left(S, \mathcal{I}_{Z}(u, v)\right)=(u+1)(v+1)-3 a$ and $u \geq 2$, we get a contradiction and hence prove the First Claim.

Second Claim: $h^{1}\left(S, \mathcal{I}_{Z^{\prime \prime}}(u, v)\right)=0$.

Proof of the Second Claim: Here we use the assumption $(u+1)(v+1)-3 a \geq 4$ to get $h^{0}\left(S, \mathcal{I}_{Z^{\prime}}(u, v)\right)=0$. Then we may easily adapt the proof of the First Claim to get the Second Claim.

By the Second Claim we get that for a general $C \in\left|\mathcal{I}_{Z}(u, v)\right|$ the morphism $\pi_{1} \circ \nu$ has at least one ordinary ramification point which is the only ramification point in its fiber of $\pi_{1} \circ \nu$.

Proof of Theorem 2. Set $u:=m k$. Assume the existence of a degree $k$ multiple covering $f: X \rightarrow Y$ of a smooth curve of genus $q$ such that there is $R \in \operatorname{Pic}(Y)$ with $\left.L:=\nu^{*}\left(\mathcal{O}_{C}(1,0)\right) \cong f^{*}(R)\right)$ and $h^{0}\left(X, \nu^{*}\left(\mathcal{O}_{C}(1,0)\right)\right)=h^{0}(Y, R)$. Fix any integer $t$ such that $1 \leq t \leq v-2$. By the last part of Example 1 we have $h^{0}\left(X, L^{\otimes t}\right)=(t+1)+h^{\overline{1}}\left(S, \overline{\mathcal{I}}_{Z}(u, v-2-t)\right)$. Notice that $h^{0}\left(X, L^{\otimes t}\right) \geq$ $h^{0}\left(Y, R^{\otimes t}\right) \geq t m+1-m$ and that $t m+1-q \geq t-2$ if $m(t-1) \geq q+1$. Hence $h^{1}\left(S, \mathcal{I}_{Z}(u-2, v-2-t)\right) \neq 0$ if $m(t-1) \geq q+1$. We have $h^{1}\left(S, \mathcal{I}_{Z}(u-2, v-2-t)\right)=0$, if $a \leq \min \{u-1, v-1-t\}$. 


\section{REFERENCES}

[1] E. Arbarello and M. Cornalba, Footnotes to a paper of Beniamino Segre, Math. Ann. 256 (1981), no. 3, 341-362.

Received: September 4, 2005 